\title{
Upper bounds for perfect matchings in Pfaffian and planar graphs
}

\author{
Afshin Behmaram* \\ School of Mathematics, Statistics and Computer Science \\ University of Tehran \\ Tehran, Iran \\ behmaram@ut.ac.ir \\ Shmuel Friedland ${ }^{\dagger}$ \\ Department of Mathematics, Statistics and Computer Science \\ University of Illinois at Chicago \\ Chicago, Illinois 60607-7045, USA \\ friedlan@uic.ed \\ Submitted: Oct 26, 2012; Accepted: Mar 13, 2012; Published: Mar 24, 2013 \\ Mathematics Subject Classifications: 05C88, 05C89
}

\begin{abstract}
We give upper bounds on weighted perfect matchings in Pfaffian graphs. These upper bounds are better than generalized Bregman's inequality. We show that some of our upper bounds are sharp for 3 and 4-regular Pfaffian graphs. We apply our results to fullerene graphs.
\end{abstract}

Keywords: Perfect matchings, Pfaffian graphs, fullerene graphs, Hadamard-Fischer determinant inequality.

\section{Introduction}

The aim of this paper is give upper bounds on the number of matchings in Pfaffian graphs using the Hadamard-Fischer determinant inequality. Let $G=(V, E)$ be a simple undirected graphs with the sets of $V$ vertices and $E$ edges. Denote by $d(v)$ the degree of $v \in V ; \Delta(G):=\max \{d(v), v \in V\}$; perfmat $G$ the number of perfect matches in $G ; K_{n}$ a complete graph on $n$ vertices; $C_{n}$ a cycle on $n$ vertices. The simplest result of this paper

*This work was supported by ministry of science of Iran and by UIC

†'This work was supported by NSF grant DMS-1216393 
can be stated as follows. Assume that $G$ is a planar graph, or more generally Pfaffian. Then

$$
\text { perfmat } G \leqslant \prod_{v \in V} d(v)^{\frac{1}{4}}
$$

We show that if $\Delta(G) \geqslant 3$ then the above inequality is stronger than the upper bound given in [1]. Next we show that the above upper bound is sharp for the following $d-$ regular connected planar graphs. For $d=3$ two planar graphs: $K_{4}$ and $C_{4} \times K_{2}$, (the direct product of $C_{4}$ and $K_{2}$ ). For $d=4$ : a unique planar graph called octahedron, (a complement of a perfect match in $K_{6}$ and hence planar). The rest of the paper is devoted to improvements of (1.1).

We survey briefly the contents of this paper. In $\S 2$ we recall the basic notions and results we use in this paper. In $\S 3$ we apply Hadamard's determinant inequality to give an estimate on the sum of weighted perfect matches in a Pfaffian graph with weighted edges. We also give upper bounds on perfect matchings in planar graphs with a planar girth $g^{\prime}$, (which is not less than the girth of the graph.) In $\S 4$ we discuss examples where the inequality (1.1) is sharp for 3 and 4-regular Pfaffian graphs. In $\S 5$ we give upper bounds on the number of perfect matchings in a Pfaffian graph $G=(V, E)$ with no 4-cycles, in terms of the maximal match in the derived graph $G^{\prime}=\left(V, E^{\prime}\right)$, where $(u, v) \in E^{\prime}$ if and only if there is a path of length 2 in $G$. In $\S 6.1$, we introduce circular and semi-circular graphs and describe some classes of fullerene, (cubic graphs with pentagon and hexagon faces), which have circular graph structure. In $\S 6.2$, the most technical section of this paper, we derive upper bounds of the number of perfect matches in semi-circular graphs with no 3 and 4-cycles. As a corollary we show that a fullerene graph $G$ nontrivial cyclic 5 -edge cut satisfies perfmat $G \leqslant 20^{\frac{|V|}{12}}$.

\section{Basic notions and results}

In this section we state our notation and recall some known results that will be used in this paper. Denote $n:=|V|$ the number of vertices; $m:=|E|$ the number of edges; $A(G)$ the adjacency matrix of $G ; K_{r, r}$ a complete bipartite $r$-regular graph on $2 r$ vertices.

Let $G^{\prime}=\left(V^{\prime}, E^{\prime}\right)$ be another graph. Then the cartesian product of the graphs $G$ and $G^{\prime}$, denoted by $G \times G^{\prime}=\left(V \times V^{\prime}, \tilde{E}\right)$, is defined as follows. $\left(\left(u, u^{\prime}\right),\left(v, v^{\prime}\right)\right) \in \tilde{E}$ if and only if the following conditions hold. Either $u=v$ and $\left(u^{\prime}, v^{\prime}\right) \in E^{\prime}$ or $u^{\prime}=v^{\prime}$ and $(u, v) \in E$.

Let $G=(V, E)$ be a simple graph. Identify $V$ with $[n]:=\{1, \ldots, n\}$. Orient each edge $e \in E$. Then the skew symmetric adjacency matrix of $S(G)=\left[s_{i j}\right]_{i, j=1}^{n}$ is defined as follows. $s_{i j}=0$ if the undirected edge $(i, j)$ is not in $E$. Assume that the undirected edge $(i, j)$ is in $E$. Then $s_{i j}=1$ if the oriented edge goes from the vertex $i$ to vertex $j$. Otherwise $s_{i j}=-1$. Note that $A(G)=\left[\left|s_{i j}\right|\right]$.

Let $n$ be even an denote $n^{\prime}:=\frac{n}{2}$. A perfect match $M$ in $G$ is a collection of $n^{\prime}$ edges in $G$ which do not have a common vertex. Denote by $\mathcal{M}(G)$ the set of perfect matches in $G$. Then perfmat $G=|\mathcal{M}(G)|$.

Let $w: E \rightarrow \mathbb{R}_{+}:=[0, \infty)$ be the weights of the edges $E$. Denote $G_{w}:=(V, E, w)$ 
the weighted graph. Then $A\left(G_{w}\right)=[w(u, v)]_{u, v \in V}$ is the weighted adjacency matrix of $G_{w}$, where $w(u, v)=0$ if the edge $(u, v) \notin E$. Denote by $d_{w}(v):=\sum_{u,(u, v) \in E} w(u, v)$ the weighted degree of $v \in V$. Direct the edges of $G$. Then the corresponding weighted sign adjacency matrix is $S\left(G_{w}\right)=[s(u, v)]_{u, v \in V}$ is a skew symmetric matrix, where $s(u, v) \geqslant 0$ if the oriented edge is from $u$ to $v$.

Let $M \in \mathcal{M}\left(K_{n}\right)$. Then $M=\left\{\left(i_{1}, j_{1}\right), \ldots,\left(i_{n^{\prime}}, j_{n^{\prime}}\right)\right\}$ where $\left\{i_{1}, j_{1}, \ldots, i_{n^{\prime}}, j_{n^{\prime}}\right\}=[n]$. We will assume that $i_{k}<j_{k}$ for $k \in\left[n^{\prime}\right]$ and $1 \leqslant i_{1}<i_{2}<\ldots<i_{n^{\prime}} \leqslant n$. Associate with $M$ the following permutation $\sigma_{M}:[n] \rightarrow[n]: 1 \rightarrow i_{1}, 2 \rightarrow j_{1}, \ldots, n-1 \rightarrow i_{n^{\prime}}, n \rightarrow j_{n^{\prime}}$.

Let $W=\left[w_{i j}\right]_{i, j=1}^{n}$ be a symmetric matrix with zero diagonal. The Haffnian of $W$ is defined as

$$
\text { haf } W=\sum_{\left\{\left(i_{1}, j_{1}\right), \ldots,\left(i_{n^{\prime}} j_{n^{\prime}}\right)\right\} \in \mathcal{M}\left(K_{n}\right)} w_{i_{1} j_{1}} \ldots w_{i_{n^{\prime}} j_{n^{\prime}}} .
$$

Clearly, the entries of $W$ can be identified with the weights of edges in $K_{n}$. Then perfmat $\left(K_{n}\right)_{w}:=$ haf $W$ is the sum of all weighted perfect matches of $\left(K_{n}\right)_{w}$. Assume that $w_{i j}=0$ if $(i, j)$ is not an edge in $E$. Then $W$ represents $G_{w}$ and perfmat $G_{w}:=$ haf $W$ is the sum of all weighted perfect matches of $G_{w}$. $S:$

Let $S=\left[s_{i j}\right]_{i, j=1}^{n}$ be a skew symmetric matrix. Recall the definition of the Pfaffian of

$$
\text { pfaf } S=\sum_{\left\{\left(i_{1}, j_{1}\right), \ldots,\left(i_{n^{\prime}} j_{n^{\prime}}\right)\right\} \in \mathcal{M}\left(K_{n}\right)} \operatorname{sign}\left(\sigma_{M}\right) s_{i_{1} j_{1}} \ldots s_{i_{n^{\prime}} j_{n^{\prime}}} .
$$

Recall the well known formula $\operatorname{det} S=(\text { pfaf } S)^{2}$. We now state a well known result and bring its proof for completeness.

Lemma 1. Let $G=(V, E)$ be a simple graph on even number of vertices. Let $w: E \rightarrow$ $(0, \infty)$. Then

$$
\operatorname{det} S\left(G_{w}\right) \leqslant\left(\text { perfmat } G_{w}\right)^{2} \text {. }
$$

Equality holds if and only if $\operatorname{sign}\left(\sigma_{M}\right)=\operatorname{sign}\left(\sigma_{M^{\prime}}\right)$ for any $M, M^{\prime} \in \mathcal{M}(G)$.

Proof. Clearly $\mid$ pfaf $S\left(G_{w}\right) \mid \leqslant$ perfmat $G_{w}$. Since each edge in $G$ has a positive weight it follows that equality holds if and only if $\operatorname{sign}\left(\sigma_{M}\right)=\operatorname{sign}\left(\sigma_{M^{\prime}}\right)$ for any $M, M^{\prime} \in \mathcal{M}(G)$. As det $S\left(G_{w}\right)=\left(\operatorname{pfaf} S\left(G_{w}\right)\right)^{2}$ we deduce the lemma.

A graph is called Pfaffian if there is an orientation of $E$ such that $\operatorname{det} S(G)=$ (perfmat $G)^{2}$. We call such orientation a Pfaffian orientation. It was shown by Kasteleyn [10] that every planar graph is Pfaffian. Recall that an orientation of $E$ is Pfaffian if and only if the following condition hold. Let $C$ be an even cycle of $G$. If the subgraph $G \backslash C$ has a perfect matching then $C$ has an odd number of edges directed in either direction of the cycle. An example of a Pfaffian nonplanar graph is $K_{4} \times K_{2}$. See [19] for a recent review on Pfaffian graphs. Assume that $G$ is Pfaffian. It is straightforward to see that for a Pfaffian orientation and any $w: E \rightarrow[0, \infty)$ we have equality in (2.3).

Let $B=\left[b_{u v}\right]_{u, v \in V}$ be a real symmetric nonnegative definite matrix of order $|V|$, which is denoted by $B \succeq 0$. The generalized Hadamard-Fischer inequality, abbreviated here as H-F inequality, states

$$
\operatorname{det} B[U \cup W] \operatorname{det} B[U \cap W] \leqslant \operatorname{det} B[U] \operatorname{det} B[W] \text { where } U, W \subset V \text {. }
$$


Here $\operatorname{det} B[\emptyset]=1$. (See for example [8] and references therein.) Assume that $U \cap W=\emptyset$. Furthermore if the left hand-side of $(2.4)$ is positive then equality holds if and only if $B[U \cup W]$ is a block diagonal matrix $\operatorname{diag}(B[U], B[W])$. The above inequality for $U \cap W=\emptyset$ is equivalent to the Hadamard-Fischer inequality.

$$
\operatorname{det} B \leqslant \prod_{i=1}^{l} \operatorname{det} B\left[U_{i}\right]
$$

where $\cup_{i=1}^{l} U_{l}$ is a partition of $V$ into a union of nonempty disjoint sets. In particular Hadamard's determinant inequality for $B \succeq 0$ is

$$
\operatorname{det} B \leqslant \prod_{v \in V} b_{v v}
$$

If all diagonal entries of $B$ are positive then equality holds if and only if $B$ is a diagonal matrix.

We apply our result for fullerene graphs. Fullerenes are famous chemical graphs that were introduced by Harold Kroto, Richard Smalley and Robert Curl in 1985 and in 1996. The Nobel prize of Chemistry was awarded to them for discovery of fullerenes. Fullerene graph is a planar cubic graph whose faces are pentagons or hexagons. Let $F_{n}$ be a fullerene with $\mathrm{n}$ vertices. The smallest fullerene is $F_{20}$ is called dodecahedron, which consist of twelve pentagons and no hexagon. Let $\mathrm{p}$ and $\mathrm{h}$ be the number of pentagons and hexagons in fullerene graph with $\mathrm{n}$ vertices and $\mathrm{m}$ edges. By Euler's formula we have: $p=12, n=2 h+20, m=3 h+30$.

Fullerenes are a special case of planar bridgeless graphs. Recall the Lovász-Plummer conjecture which claims that perfmat $G$ is exponential in $n$ for every cubic bridgeless graph [17, Conjecture 8.1.8]. It is a generalization of the Erdős-Rényi conjecture for 3-regular bipartite graphs [4], which was proved independently by Friedland [7] and Voorhoeve [20]. The Lovász-Plummer conjecture for planar graphs was proved by Chudnovsky and Seymour [3], (the preprint was available in 2008), and the complete conjecture was demonstrated by Esperet-Kardos-King-Kral-Norine [5]. A stronger lower bound for the number of perfect matchings in fullerenes are given in [11].

\section{Upper bounds on perfect matchings in Pfaffian graphs}

Theorem 2. Assume that $n$ is even, $G$ has a Pfaffian orientation and $w: E \rightarrow[0, \infty)$. Then

$$
\text { perfmat } G_{w} \leqslant \prod_{v \in V}\left(d_{w, 2}(v)\right)^{\frac{1}{4}}, \quad d_{w, 2}(v):=\sum_{u,(u, v) \in E} w(u, v)^{2} .
$$

In particular (1.1) holds. 
Proof. Assume that $B=S\left(G_{w}\right) S\left(G_{w}\right)^{\top}=-S\left(G_{w}\right)^{2}$. Note that $B[\{v\}]=d_{w, 2}(v)$. Hadamard's determinant inequality yields that $\operatorname{det} B \leqslant \prod_{v \in V} d_{w, 2}(v)$ and $\operatorname{det} B=\operatorname{det}(S(G))^{2}=$ (perfmat $\left.G_{w}\right)^{4}$. This relation yields the theorem.

We next recall the upper bound on then number of perfect matchings in $G$ given in [1], which was mentioned in the Abstract as generalized Bregman's inequality.

$$
\text { perfmat } G \leqslant \prod_{v \in V}(d(v) !)^{\frac{1}{2 d(v)}} .
$$

Equality holds if and only if $G$ is a union of complete regular bipartite graphs.

Clearly $(d !)^{\frac{1}{2 d}}=d^{\frac{1}{4}}$ for $d=1,2$. For an integer $d \geqslant 3$ we have the following inequality.

\section{Lemma 3.}

$$
(d !)^{\frac{1}{2 d}}>d^{\frac{1}{4}} \quad \text { for } d=3, \ldots
$$

Proof. Stirling's formula for $d$ ! implies the inequality $(d !)^{\frac{1}{d}} \geqslant \frac{d}{e}$. Next observe that $\sqrt{d}<\frac{d}{e}$ for $d \geqslant 8$. One easily verifies that (3.3) holds for $d=3, \ldots, 7$.

As perfmat $K_{r, r}=r$ ! we deduce a well known result.

Corollary 4. $K_{r, r}$ is not Pfaffian for $r \geqslant 3$.

Recall that the girth $g(G)$ of a graph $G$ is the length of the shortest cycle in $G$. Let $G$ be a planar connected graph which is not a tree. We define a planar girth of $G$ as follows. Assume that we have a realization of $G$ as a partition of the plane to $f$ faces $F_{1}, \ldots, F_{f}$ including the unbounded face. Denote by $\ell\left(F_{i}\right)$ the length of the face $F_{i}$. Then the maximum value of $\min \ell\left(F_{i}\right)$ for all possible realization of $G$ as a planar graph is called the planar girth of $G$, and is denoted by $g^{\prime}(G)$. A realization of $G$ where $g^{\prime}(G)$ is the minimal length of the faces is called an optimal realization. It is easy to construct an example of a connected planar graph $G$ with $g(G)=3$ and $g^{\prime}(G)>3$. Let $g^{\prime}(T)=\infty$ for a tree. For a nonconnected planar graph $G$ we define $g^{\prime}(G)$ to be the minimum of the planar girths of its connected components.

The following result is probably well known and we bring its proof for completeness.

Lemma 5. Let $G$ be a planar graph with a finite planar girth $g^{\prime}$. Then

$$
m \leqslant \frac{g^{\prime}}{g^{\prime}-2}(n-2)
$$

Equality holds if and only if $G$ is connected, and there exists an optimal realization of $G$, such that each face, including the outside face, is bounded by a cycle of length $g^{\prime} \geqslant 3$.

Proof. Assume that $G$ is connected. Let $F_{1}, \ldots, F_{f}$ be the $f$ faces of an optimal realization of $G$. Euler's formula states $f-m+n=2$. Clearly $2 m=\sum_{i=1}^{f} g\left(F_{i}\right) \geqslant g^{\prime} f=g^{\prime}(2+m-n)$, which implies (3.4). Assume that $G$ has $l$ connected components $G_{i}, i=1, \ldots, l$. Then $m_{i} \leqslant \frac{g^{\prime}}{g^{\prime}-2}\left(n_{i}-2\right)$ if $G_{i}$ has a cycle. If $G_{i}$ has no cycle, i.e. $G_{i}$ is a tree, then $m_{i}=n_{i}-1$. This implies (3.4). The equality case follows straightforward. 
Theorem 6. In every planar graphs with a finite planar girth $g^{\prime}$ the number of perfect matchings are less than $\left(\frac{2 g^{\prime}}{g^{\prime}-2}\right)^{\frac{n}{4}}$. In particular, if $g^{\prime}>3$, i.e. there is a realization of $G$ such that each face is not a triangle, then the number of perfect matchings is less than $2^{\frac{n}{2}}$.

Proof. Apply the AM-GM inequality and the fact that the sequence $\left(1-\frac{2}{l}\right)^{\frac{l}{2}}$ is an increasing sequence converging to $e^{-\frac{1}{2}}$ to deduce

$$
\text { perfmat } G \leqslant \prod_{v \in V}(d(v))^{\frac{1}{4}} \leqslant\left(\frac{2 m}{n}\right)^{\frac{n}{4}} \leqslant\left(\frac{2 g^{\prime}(n-2)}{\left(g^{\prime}-2\right) n}\right)^{\frac{n}{4}}<e^{-\frac{1}{2}}\left(\frac{2 g^{\prime}}{g^{\prime}-2}\right)^{\frac{n}{4}} .
$$

If $g^{\prime} \geqslant 4$ then $\frac{2 g^{\prime}}{g^{\prime}-2} \leqslant 4$ and the above inequality implies that perfmat $G<2^{\frac{n}{2}}$.

\section{Sharp upper bounds on 3 and 4-regular Pfaffian graphs}

Let $\phi(k, G)$ be the number of matches which cover $2 k$ vertices of $G$. Define $G \times K_{2}$, the cartesian product of $G$ and $K_{2}$, as two copies of $G$ where we join by an edge two copies of each vertex $v \in V$. It is easy to show that $C_{4} \times K_{2}$ is a planar graph. Clearly perfmat $C_{4}=2$, perfmat $K_{4}=3$. Hence

$$
\begin{aligned}
& \text { perfmat }\left(C_{4} \times K_{2}\right)=\operatorname{perfmat}\left(C_{4}\right)^{2}+\phi\left(1, C_{4}\right)+1=4+4+1=3^{2}, \\
& \text { perfmat }\left(K_{4} \times K_{2}\right)=\operatorname{perfmat}\left(K_{4}\right)^{2}+\phi\left(1, K_{4}\right)+1=9+6+1=4^{2} .
\end{aligned}
$$

It is well known that $K_{4} \times K_{2}$ is Pfaffian [19].

Theorem 7. Let $G=(V, E)$ be a 3-regular simple Pfaffian graph. Then the number of perfect matchings in $G$ is not more than $3^{\frac{|V|}{4}}$. For a planar 3-regular graph $G$ equality holds if and only if $G$ is a disjoint union of copies of $K_{4}$ or $C_{4} \times K_{2}$.

Proof. Hadamard's determinant inequality gives the upper bound. To discuss the equality case for a planar graph $G$ it is enough to show that each connected component $H$ of $G$ is either $K_{4}$ or $C_{4} \times K_{2}$. Since the graph is simple, each $H$ contains at least 4 vertices. If $H$ has 4 vertices then $H=K_{4}$. Assume to that $H=\left(V_{1}, E_{1}\right)$ contains more than 4 vertices. Since we assumed that $\operatorname{det} S(H)=3^{\frac{\left|V_{1}\right|}{2}}$ it follows that $S(H)^{2}=-3 I$, where $I$ is the identity matrix. This means that if there is a path of length two connecting two vertices $u, v \in V_{1}$ then we have exactly another path from $u$ to $v$, as $H$ is 3-regular. Consider the faces generated by the planar graph $H$. Suppose first that we have a face bounded by a triangle with vertices $u, v, w$. Since there is a path of length two between any two vertices of this triangle, each edge of this triangle must be bounded by another triangle. The assumption $H$ is 3-regular implies that $H=K_{4}$, contrary that $H$ has more than 4 vertices.

So $g^{\prime}(H) \geqslant 4$. Suppose there is a face $F_{1}$ of $H$ of which is bounded by a cycle of length 5 at least. Take three consecutive points $u_{1}-u_{2}-u_{3}$ on this cycle. Since the 
distance from $u_{1}$ to $u_{3}$ is $2 H$ contains another path of length two between $u_{1}$ and $u_{2}$, namely $u_{1}-v-u_{3}$. This path must lie on one or two adjacent faces of $H$ to $F_{1}$. Since the degree of $u_{2}$ is 3 this path lies on two adjacent triangles with vertices $u_{1}, u_{2}, v$ and $u_{2}, v, u_{3}$. This contradicts our claim that $g^{\prime}(H)>3$. Hence each face of $H$ is bounded by a 4-cycle. Take one compact face $F_{1}$ bounded by the 4 -cycle $u_{1}-u_{2}-u_{3}-u_{4}-u_{1}$. Since the degree of each vertex of $H$ is 3 there must be another adjacent face of $F_{1}$ bounded by the cycle $u_{1}-u_{2}-v_{1}-v_{2}-u_{1}$, call it $F_{2}$. Since the distance between $v_{1}$ and $u_{3}$ is 2 we must have a 4-cycle $u_{3}-u_{2}-v_{1}-v_{3}-u_{3}$ which bounds the face $F_{3}$ adjacent to $F_{1}$ and $F_{2}$. Similarly, we must have a 4 -cycle $u_{4}-u_{1}-v_{3}-v_{4}$ which bounds the face $F_{4}$ adjacent to $F_{1}$ and $F_{2}$. Note that $v_{3} \neq v_{4}$. Since the $\operatorname{dist}\left(v_{2}, v_{3}\right)=\operatorname{dist}\left(v_{1}, v_{4}\right)=2$ we must have an edge $v_{3}-v_{4}$ in $H$. Then $H=C_{4} \times K_{2}$.

The octahedron graph, denoted by $O$, is a 4-regular graph with 6 vertices. It is obtained from $K_{6}$ by deleting 3 edges forming a complete match. Hence $O$ is unique up to an isomorphism. It is easy to see that $O$ is planar. A straightforward calculation shows that perfmat $O=8$. Indeed, recall that perfmat $K_{6}=15$. Take a perfect match $M$ in $K_{6}$ and count all matches in $K_{6}$ which contain at least one edge from $M$. One match is $M$. All other matches contain exactly one edge from $M$. Let $(u, v) \in M$. Then $3=$ perfmat $K_{4}$ is the number of matches in $K_{6}$ containing $(u, v) \in M$. One of them is $M$. Hence the number of all matches in $K_{6}$ which contain $(u, v)$ and no other edges from $M$ is 2 . Thus perfmat $O=15-(1+3 \times 2)=8$.

Let $G$ be a connected 4 -regular planar graph with more than 6 vertices. In $[15, \S 2]$ it is shown that every 4-regular connected planar graph $G$ on more than 6 vertices contains one of the configurations $\mathrm{A}, \mathrm{B}, \mathrm{C}$, or D. In particular, it follows that not all the faces of $G$ are triangles.

Theorem 8. Let $G=(V, E)$ be a 4-regular simple Pfaffian graph on an even number of vertices. Then the number of perfect matchings is not more than $4^{\frac{|V|}{4}}$. For planar graphs equality holds if and only if $G$ is a disjoint union of copies the octahedron graph.

Proof. The inequality perfmat $G \leqslant 2^{\frac{n}{2}}$ follows from Hadamard's determinant inequality. Equality holds if and only if $S(G)^{2}=-4 I$. As the number of perfect matches in $O$ is 2 to the power of a half of the vertices we deduce that $S(O)^{2}=-4 I_{6}$.

Let $G$ be a 4-regular planar graph which satisfies $S(G)^{2}=-4 I$. The inequality (2.3) implies that the orientation inducing $S(G)$ is Pfaffian. Hence each connected component $H$ of $G$ satisfies $S(H)^{2}=-4 I$. Furthermore $S(H)$ is a Pfaffian orientation of $H$ and perfmat $H=2^{\frac{\left|V_{1}\right|}{2}}$. Assume to the contrary that $H=\left(V_{1}, E_{1}\right)$ is not $O$, i.e. $\left|V_{1}\right| \geqslant 8$.

Observe next that the equality $S(H)^{2}=-4 I$ implies the following condition. Assume that there is a path of length two connecting two vertices $u, v \in V_{1}$. Then we have either one or three additional paths of length two from $u$ to $v$, as $H$ is 4-regular. Theorem 6 implies that $g^{\prime}(H)=3$. Take a face $F_{1}$ of $H$ which is a triangle which vertices $v_{1}, v_{2}, v_{3}$. As in the proof of Theorem 7 each neighboring face $F_{1}$ is a triangle. Hence all faces of $H$ are triangles. This contradicts the theorem of [15]. 
Note that $K_{4} \times K_{2}$ is a 4-regular connected nonplanar Pfaffian graph for which the number of matchings is $4 \frac{|V|}{4}$. We do not know of other examples of 4-regular connected nonplanar Pfaffian graph for which the number of matchings is $4^{\frac{|V|}{4}}$.

\section{Applications of $\mathrm{H}-\mathrm{F}$ inequality to Pfaffian graphs}

Theorem 9. Let $G=(V, E)$ be a Pfaffian connected graph. Denote by $G^{\prime}=\left(V, E^{\prime}\right)$ be the induced graph by $G$, where $(u, v) \in E^{\prime}$ if and only if there exists a path of length 2 between $u$ and $v$ in $G$. Let $M^{\prime}$ be a match in $G^{\prime}$. Assume that $G$ does not have 4-cycles. Then

$$
\text { perfmat } G \leqslant\left(\prod_{(u, v) \in M^{\prime}}(d(u) d(v)-1)\right)^{\frac{1}{4}}\left(\prod_{v \in V \backslash V\left(M^{\prime}\right)} d(v)\right)^{\frac{1}{4}} .
$$

Proof. Consider the matrix $B=-S(G)^{2} \succeq 0$. Let $B=-S(G)^{2}=\left[b_{u v}\right]_{u, v \in V}$. Assume that $u \neq v$. The assumption that $B$ has no 4 -cycles means that $b_{u v}= \pm 1$ if there exists a path of length 2 between $u$ and $v$ and otherwise $b_{u v}=0$. Clearly, $b_{u u}=d(u)$. Associate with $M^{\prime}$ the following partition of $[n]$. With each pair $(u, v) \in M^{\prime}$ associate $J_{u, v}:=\{u, v\}$. For all vertices $w \in V$ not covered by $M^{\prime}$ we associate $J_{w}=\{w\}$. Apply (2.5) to deduce (5.1).

One can use the following proposition to estimate $\left|M^{\prime}\right|$.

Proposition 10. Assume that $G=(V, E), n=|V|$ is a connected graph with a path of length $l \geqslant 3$. Then the induced graph $G^{\prime}$ given in Theorem 9 has a match $M^{\prime}$ of cardinality $2\left\lfloor\frac{l}{4}\right\rfloor$ and $\left\lfloor\frac{l}{2}\right\rfloor$ if $l$ is even or odd respectively.

It is conjectured that any fullerene graph has a hamiltonian cycle. It is known that any fullerene graph has a cycle of length $\frac{5 n-4}{6}$ at least [13].

Corollary 11. Assume that $G=(V, E)$ is a fullerene graph. Then perfmat $G$ is bounded above by the following quantities.

$$
\left\{\begin{array}{l}
8^{\frac{n}{8}} \text { if } G \text { has a hamiltonian cycle and } n \text { is divisible by } 4 \\
8^{\frac{n-2}{8}} \sqrt{3} \text { if } G \text { has a hamiltonian cycle and } n \text { is not divisible by } 4 \\
8^{\frac{1}{4}\left\lfloor\frac{5 n-4}{12}\right\rfloor} 3^{\frac{n-2\left\lfloor\frac{5 n-4}{4}\right\rfloor}{4}} .
\end{array}\right.
$$

Theorem 12. Every cubic Pfaffian graph with $n$ vertices, which has a perfect match and no 4-cycles, has at most $8 \frac{n}{12} 3^{\frac{n}{12}}$ perfect matching.

Proof. Let $M$ be a perfect match in $G$. Then the complement of $M$ in $G$ is a union of spanning cycles $C_{p_{1}}, \ldots, C_{p_{k}}$. Each even cycle $C_{p_{i}}$ induces a match of order $2\left\lfloor\frac{p_{i}}{4}\right\rfloor$ in the induced graph $G^{\prime}$. Each odd cycle $C_{p_{j}}$ induces a match of order $\frac{p_{j}-1}{2}$ in the induced graph $G^{\prime}$. Let $M^{\prime}$ be a match in $G^{\prime}$ induced as above by the cycles $C_{p_{1}}, \ldots, C_{p_{k}}$. The smallest $M^{\prime}$ will be obtained when each $p_{i}=3$. So $\left|M^{\prime}\right| \geqslant \frac{n}{3}$. Apply Theorem 5.1 to deduce the theorem. 


\section{Circular and semi-circular graphs}

\subsection{Definition and properties}

A connected planar graph $G=(V, E)$ is called semi-circular if $G$ can be obtained as follows. Draw $k \geqslant 1$ circles $O_{1}, \ldots, O_{k}$ in the plane of radii $0<r_{1}<\ldots<r_{k}$ centered at the origin. At each circle $j$ we assume that we have $m_{j} \geqslant 3$ vertices for $j=1, \ldots, k$, denoted as $U_{j} \subset V$. The subgraph of $G\left(U_{j}\right)$ is the cycle $C_{m_{j}}$ given by $O_{j}$ and the vertices $U_{j}$ for $j \in[k]$. In addition connect some vertices in two adjacent circles $U_{i}$ and $U_{i+1}$ such that the corresponding edges lying in the annulus $r_{i} \leqslant \sqrt{x^{2}+y^{2}} \leqslant r_{i+1}$ and do not intersect in the interior $r_{i}<\sqrt{x^{2}+y^{2}}<r_{i+1}$, for $i=1, \ldots, k-1$. The rest of the vertices $V_{0}$ of $G$ lie inside the open disk $x^{2}+y^{2}<r_{1}$. So $G\left(U_{1} \cup V_{0}\right)$ is a connected planar graph whose vertices and edges lie in the closed disk $x^{2}+y^{2} \leqslant r_{1}$. (Since $G$ is connected we assume that we have at least one edge connecting the sets $V_{i}$ and $V_{i+1}$ for $i \in[k-1]$.) A semi-circular graph is called circular if $V_{0}=\emptyset$.

The following characterization of a semi-circular graph is straightforward.

Lemma 13. A connected planar graph $G$ is semi-circular if and and only if it can be realized in the plane as follows. Assume that $G=(V, E)$ induces $f-1$ bounded faces $F_{1}, \ldots, F_{f-1}$ and an unbounded face $F_{f}$ in $\mathbb{R}^{2}$. Then there exists a following sequence of subgraphs of $G$ : $G=G_{k}=\left(V_{k}, E_{k}\right) \supsetneqq G_{k-1}=\left(V_{k-1}, E_{k-1}\right) \supsetneqq \ldots \supsetneqq G_{1}=\left(V_{1}, E_{1}\right)$ with the following properties. The bounded faces of each $G_{i}$ are $F_{j}, j \in \mathcal{A}_{i}$, where $\mathcal{A}_{k}=[f-1] \supsetneqq$ $\mathcal{A}_{k-1} \supsetneqq \ldots \supsetneqq \mathcal{A}_{1} \neq \emptyset$. The unbounded face of $G_{i}$ is a cycle $C_{m_{i}}$, where $m_{i} \geqslant 3$. $V_{i-1}$ are all the vertices of $V$ contained in the interior of $\cup_{j \in \mathcal{A}_{i}} F_{j}$, for $j=k, \ldots, 1$. Furthermore $G$ is circular if $V_{0}=\emptyset$.

Clearly, any connected planar graph with edges and no bridges is a semi-circular graph with $k=1$. So a really interesting semi-circular graphs is a graph where $V_{0}$ is a relatively small set in $V$. Available representations of 26,70 fullerene graphs are only semi-circular with a relative small $V_{0}$. The 20,24,60 fullerene graphs are circular graphs. Figure 1 shows the circular representation of $C_{20}$ and $C_{60}$.
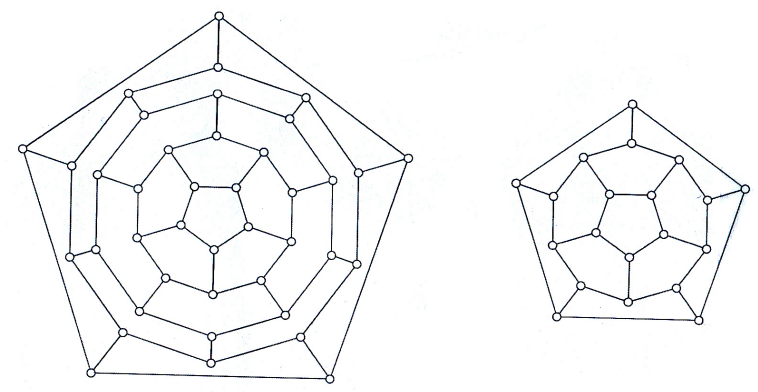

Figure 1: Circular representation of fullerenes $C_{60}$ and $C_{20}$.

We now define two infinite families of circular fullerene graphs see [16, 8-4]. The first circle and the last circle is $C_{k}$ with $k=5$ or $k=6$. I.e. each circle bounds a bounded 
and unbounded either pentagon or hexagon face respectively. All other $\ell$ circles, $\ell \geqslant 1$, are $C_{2 k}$. That is, the first and the last $C_{k}$ are surrounded by layers of $k$ pentagons. Note that the number of vertices in such fullerene graph is $2 k(\ell+1)$. We call each family of these fullerene graphs pentacap and hexacap for $k=5,6$ respectively. In $[12,14]$ it was proved that a fullerene graph with a non trivial 5 -edge cut is a pentacap with $l \geqslant 2$.

We observe that we can slightly generalize the construction of pentacaps and hexacaps fullerenes.

Proposition 14. Let $F=(V, E)$ be a circular fullerene graph where the first circle is $C_{k}$ and the second circle is $C_{2 k}$ for $k=5,6$. (I.e. the first circle is surrounded by a layer of $k$ pentagons.) Then there exists a circular fullerene graph $F^{\prime}=\left(V^{\prime}, E^{\prime}\right)$ with $\left|V^{\prime}\right|=|V|+k+1$.

Proof. Add $k$ vertices, $u_{1}, \ldots, u_{k}$ to the first circle $C_{k}$, one new between two old. Add a new inner circle $C_{k}$ with vertices $w_{1}, \ldots, w_{k}$ and connect $u_{i}$ with $w_{i}$ for $i=1, \ldots, k$.

Recall the leapfrog operation on a simple connected bridgeless planar graph $G=(V, E)$ with faces $F_{1}, \ldots, F_{f}$, including the unbounded face. Choose a point $w_{i}$ in the interior of each $F_{i}$. Let $W=\left\{w_{1}, \ldots, w_{f}\right\}$. Then $\tilde{G}=(\tilde{V}, \tilde{E})$ where $\tilde{V}=V \cup W$ and $\tilde{E}=E \cup E_{1}$. The edges $E_{1}$ are obtained by connecting each $w_{i}$ to each vertex $V$ on the boundary of $F_{i}$. Since $G$ is bridgeless it follows that $\left|E_{1}\right|=2|V|$. Note that all faces $\tilde{F}_{1}, \ldots, \tilde{F}_{\tilde{f}}$ of $\tilde{G}$ are triangles. Then $L e(G)=\left(V^{\prime}, E^{\prime}\right)$ is a connected planar graph, where $\left|V^{\prime}\right|=|\tilde{f}|$. Each vertex $v_{i}^{\prime} \in V^{\prime}$ is an interior point of $\tilde{F}_{i} . \quad\left(v_{i}^{\prime}, v_{j}^{\prime}\right) \in E^{\prime}$ if and only if $\tilde{F}_{i}$ and $\tilde{F}_{j}^{\prime}$ have a common edge. So $\operatorname{Le}(G)$ is the Poincaré dual of $\tilde{G}$. It is known that if $G$ is fullerene then $L e(G)$ is fullerene [6]. Figure 2 shows the fullerene $C_{20}$ and its leapfrog $C_{60}$
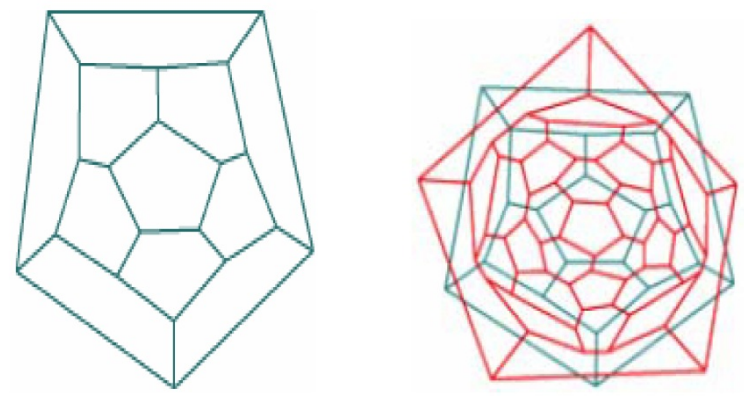

Figure 2: Leapfrog of fullerene $C_{20}, l e\left(C_{20}\right)=C_{60}$.

Theorem 15. Let $F$ be a pentacap or hexacap. That is $F$ is a circular fullerene with inner and outer circles $C_{k}, k=5,6$ and $\ell$ circles $C_{2 k}$. Then $L e(F)$ is a circular fullerene if and only if $\ell$ is odd. Furthermore, if $\ell$ is odd then the circular structure of $L e(F)$ is as follows. The first inner circle is $C_{k}$, the second circle is $C_{3 k}$, then there are $\frac{3 \ell-1}{2}$ circles $C_{4 k}$, then follows the circle $C_{3 k}$, and the last circle is $C_{k}$. If $\ell$ is even then Le $(F)$ has the following semi-circular structure. The first circle is $C_{k}$ then the next $\frac{3 l}{2}$ circles are $C_{4 k}$. The circle number $\frac{3 \ell}{2}+1$ is $C_{3 k}$. The last circle is $C_{k}$. Inside the first circle there are $k$ vertices. 
Proof. We identify the vertices of $L e(F)$ with the triangle faces of $\tilde{F}$. The $k$ triangles inside the first circle $C_{k}$ are denoted by $\alpha_{1}$ and the $k$ triangles outside the last circle of $C_{k}$ of $F$ are denoted by $\alpha_{2}$. Consider first the $k$ pentagons surrounding the first circle in $F$. Each such a pentagon is divided to 5 triangles in $\tilde{F}$. The triangle which has one edge on the first circle we name $a_{1}$. The two neighboring triangles of $a_{1}$ in this pentagon are called $b_{1}$. The other two triangles of this pentagon are called $c_{1}$. Each $c_{1}$ has one edge on the second circle of $F$. The names of the triangles of each of the $k$ pentagons surrounding the last circle of $F$ is called similarly $a_{2}, b_{2}, c_{2}$. So each $\alpha_{i}$ has the following 3 neighbors: two $\alpha_{i}$ 's and one $a_{i}$. The neighbors of $a_{i}$ are $\alpha_{i}$ and two $b_{i}$ 's. The neighbors of $b_{i}$ are $a_{i}, b_{i}, c_{i}$.

Next we divide each hexagon to six triangles of the types $A, B, C$. The two $A$ 's have one edge on a circle $p$ or $p=2, \ldots, \ell-1$. Then we have two triangles $B$. Note that the interior of each edge of $B$ lies in the interior of the hexagon. Then we have two triangles $C$. Each $C$ have one edge on the circle $p+1$ of $F$. Assume for simplicity of the argument that $\ell \geqslant 4$, i.e. we have at least three layer of hexagons. So the neighbors of $c_{1}$ are $b_{1}, c_{1}, A$. The neighbors of $c_{2}$ are $b_{2}, c_{2}, C$.

We now analyze the neighbors of the six triangles induced by hexagons. First, let us consider the first layer of hexagons between the second and the third layer of $F$. The neighbors of $A$ are $c_{1}, A, B$. The neighbors of $B$ are $A, B, C$. (This fact holds for any hexagon.) The neighbors of $C$ are $B, C$ located inside the the same hexagon and a triangle $A$ locates in the next level of hexagons, between the third and the fourth circles. (This fact holds for all layers of hexagons except the last layer located between the circle $\ell$ and $\ell+1$ of $F$.) For all other hexagons located between the circle $p$ and $p+1$ of $F$ for $p=3, \ldots, \ell+1$ the neighbors of $A$ are $A, B, C$. Finally, in the last layer of hexagons, the neighbors of $C$ are $B, C, c_{2}$.

We now discuss the semi-circular structure of $L e(F)$. We assume that we have $q$ circles. The last circle $q$ of $L e(F)$ is $C_{k}$, composed of $k \alpha_{2}$. The circle $q-1$ is $C_{3 k}$ of the form $a_{2}-b_{2}-b_{2}-a_{2}-b_{2}-b_{2}-\ldots-b_{2}-b_{2}-a_{2}$. The circle $q-2$ is $C_{4 k}$ of the form $c_{2}-c_{2}-C-C-c_{2}-c_{2}-\ldots-C-C-c_{2}-c_{2}$. The $q-3$ circle is $C_{4 k}$ of the form is $B-B-A-A-\ldots-A-A-B-B$, since it uses 4 triangles in each hexagon in the last hexagon level. The $q-4$ circle of $L e(F)$ is $C_{4 k}$ of the form $B-C-C-B-B-C-C-B-\ldots-B$, consisting of triangles obtained from the hexagons in the level between the circles $\ell-1$ and $\ell$ of $F$. The circle $q-5$ of $L e(F)$ is $A-A-C-C-\ldots-A-A$, where the triangles $A$ are in the previous level and the triangles $C$ are from the next level of hexagons. The $q-6$ circle is $C_{4 k}$ of the form $B-C-C-B-B-C-C-B-\ldots-B$.

Suppose first that $\ell$ is odd. Then it is straightforward to see that the third circle of $L e(F)$ is $C_{4 k}$ of the from $A-A-c_{1}-c_{1}-A-A-\ldots-A-A$. The second circle of $L e(F)$ is $C_{3 k}$ of the form $a_{1}-b_{1}-b_{1}-a_{1}-\ldots-a_{1}$. The first circle of $L e(F)$ is $C_{k}$ consisting of $\alpha_{1}$ triangles. So the circular structure of $L e(F)$ is $C_{k}-C_{3 k}-C_{4 k}-\ldots-C_{4 k}-C_{3 k}-C_{k}$. Since the number of circles is $q$ the total number of vertices is $4 k(q-4)+8 k$. Since $F$ had $(\ell+1) 2 k$ vertices we deduce that $3(\ell+1) 2 k=4 k(q-2)$. So $q=\frac{3 \ell-1}{2}+4$ circles. So we have exactly $\frac{3 \ell-1}{2}$ circles $C_{4 k}$. These arguments apply also to the case $\ell=1,3$. 
Assume that $\ell$ is even. By the above method the last circle is $C_{k}$. One before the last circle is a circle $C_{3 k}$ of the form $A-B-B-A-\ldots-A$. Then follows a sequence of circles $C_{4 k}$. Finally the first cycle is $C_{k}$. Inside the circle one we have $k$ triangles of the form $\alpha_{1}$ and $a_{1}$. So the circles of $L e(F)$ are $C_{k}-C_{4 k}-C_{4 k}-\ldots-C_{4 k}-C_{3 k}-C_{k}$. The total number of vertices of $L e(F)$ is $(q-3) 4 k+3 k+2 k+k$. Hence $(q-3) 4 k+6 k=3(l+1) 2 k$. So $L e(F)$ has $\frac{3 l}{2} C_{4 k}$ circles.

\subsection{Upper matching estimates on semi-circular graphs}

So we can apply the generalized Hadamard's determinant inequality (2.4) to a semicircular graph $G=(V, E)$ by considering the partitioning set of edges of $V$ to $V_{1} \cup_{j \in[k-1]}$ $V_{j+1} \backslash V_{j}$. To obtain some better upper inequalities we need several auxiliary results. We start with the following lemma which is deduced straightforward.

Lemma 16. Let $S\left(C_{n}\right)$ be an orientation of $n$-cycle $C_{n}=([n], E)$ with edges $1-2-\ldots-$ $n-1$. Then there exists a diagonal matrix $D$ whose diagonal entries are \pm 1 such that $D S\left(C_{n}\right) D$ corresponds to the following orientation $1 \rightarrow 2 \rightarrow \ldots \rightarrow n$ of these $n-1$ edges in $C_{n}$. Let $T_{n,+}, T_{n,-}$ be the skew symmetric matrices corresponding to the orientation $1 \rightarrow 2 \rightarrow \ldots \rightarrow n$ and $1 \rightarrow n, n \rightarrow 1$ of $C_{n}$ respectively.

Note that

$$
\begin{cases}\operatorname{det} T_{n,+}=\operatorname{det} T_{n,-}=0 & \text { if } n \text { odd } \\ \operatorname{det} T_{n,+}=4, \quad \operatorname{det} T_{n,-}=0 & \text { if } n \text { even }\end{cases}
$$

Indeed if $n$ is odd then the determinant of any skew symmetric matrix is zero. Assume that $n$ is even. Then $\operatorname{det} T_{n,-}=0$ since $T_{n,-} \mathbf{1}=\mathbf{0}$, where $\mathbf{1}=(1, \ldots, 1)^{\top} . T_{n,+}$ represents a Pfaffian orientation of $C_{n}$. Hence $\operatorname{det} T_{n,+}$ is the square of the perfect matches in $C_{n}$, which is $2^{2}$.

Let $G=(V, E)$ be a simple graph. Let $W \subset V,|W| \geqslant 3$ and assume that the subgraph $G(W)$ is a cycle. Let $W=\left\{w_{1}, \ldots, w_{|W|}\right\}$ where the edges of $G(W)$ are $w_{1}-w_{2}-\ldots-w_{|W|}-w_{1}$. Then $D_{c}(W)$ is a diagonal matrix of order $|W|$ of the form $\operatorname{diag}\left(d\left(w_{1}\right), \ldots, d\left(w_{|W|}\right)\right)$.

Proposition 17. Let $G=(V, E)$ be a connected semi-circular graph with $k>1$ as described in Lemma 13. Let $S(G)$ be a Pfaffian orientation of $G$. Assume that $B=$ $-S(G)^{2}$. Then

$$
\text { perfmat } G \leqslant\left(\operatorname{det} B\left[V_{1}\right]\right)^{\frac{1}{4}} \prod_{i \in[k-1]}\left(\operatorname{det} B\left[V_{i+1} \backslash V_{i}\right]\right)^{\frac{1}{4}} \text {. }
$$

Assume furthermore the following conditions. First, $G$ does not have 3 and 4-cycles. (In particular, each $m_{i}:=\left|V_{i} \backslash V_{i-1}\right|>4$ for $i \in[k]$.) Second, each $u \in V_{i} \backslash V_{i-1}$ is connected to at most one vertex in $V_{i-1} \backslash V_{i-2}$ for each $i>1$ and at most to one vertex in $V_{i+1} \backslash V_{i}$ 
for each $i \in[k]$, where $V_{k+1}=V_{k}$. Then the number of perfect matchings in $G$ is not more than

$$
\begin{aligned}
& \left(\operatorname{det} B\left[V_{1}\right]\right)^{\frac{1}{4}} \times \\
& \prod_{i=2}^{k}\left(\max \left(\operatorname{det}\left(D_{c}\left(V_{i} \backslash V_{i-1}\right)-2 I_{m_{i}}-T_{m_{i},+}^{2}\right), \operatorname{det}\left(D_{c}\left(V_{i} \backslash V_{i-1}\right)-2 I_{m_{i}}-T_{m_{i},-}^{2}\right)\right)^{\frac{1}{4}} .\right.
\end{aligned}
$$

Proof. Clearly (6.2) follows from (2.4). Assume now that $G$ satisfies the additional conditions of the theorem. Let $B=-S(G)^{2}=\left[b_{u v}\right]_{u, v \in V}$. Then the diagonal entries $b_{u u}=d(u)$. Consider the off-diagonal entries of $B$. Note that $b_{u v}=0$ if there is no path of length two between $u$ and $v$ in $G$. Assume that there is a path of length two between $u$ and $v$ Since $G$ does not have a 4-cycle the path between $u$ and $v$ of length two is unique. Hence $b_{u v}= \pm 1$. Take any two points $u, v \in V_{i} \backslash V_{i-1}$. The assumptions of the theorem yield that there is a path of length two between $u$ and $v$ is two if and only if the distance between $u$ and $v$ on the cycle $G\left(V_{i} \backslash V_{i-1}\right)$ is two for $i>1$. Hence, $B\left[V_{i} \backslash V_{i-1}\right]=D_{c}\left(V_{i} \backslash V_{i-1}\right)-2 I_{m_{i}}-S^{2}\left(G\left(V_{i} \backslash V_{i-1}\right)\right)$, where the orientation of $G\left(V_{i} \backslash V_{i-1}\right)$ is induced by the orientation $G$. Recall that there exists a diagonal matrix $D_{i}$ with entries \pm 1 such that $D_{i} S\left(G\left(V_{i} \backslash V_{i-1}\right)\right) D_{i} \in\left\{T_{m_{i},+}, T_{m_{i},-}\right\}$. Clearly $D_{i} D_{c}\left(V_{i} \backslash V_{i-1}\right) D_{i}=D_{c}\left(V_{i} \backslash V_{i-1}\right)$. Hence

$\operatorname{det} B\left[V_{i} \backslash V_{i-1}\right] \leqslant \max \left(\operatorname{det}\left(D_{c}\left(V_{i} \backslash V_{i-1}\right)-2 I_{m_{i}}-T_{m_{i},+}^{2}\right), \operatorname{det}\left(D_{c}\left(V_{i} \backslash V_{i-1}\right)-2 I_{m_{i}}-T_{m_{i},-}^{2}\right)\right.$.

We now apply the above result to semi-circular cubic graphs satisfying all the above conditions. Then $D_{c}\left(V_{i} \backslash V_{i-1}\right)-2 I_{m_{i}}=I_{m_{i}}$. To estimate the right-hand side of (6.3) we need the following lemma.

Lemma 18. For $n \geqslant 3$

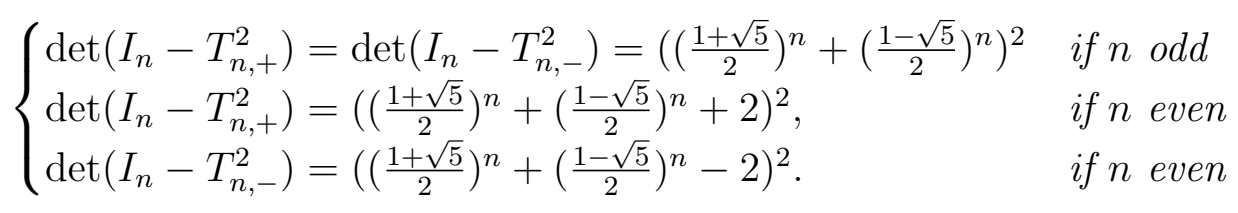

Furthermore, for $n \geqslant 3$ the subsequence $\left(\operatorname{det}\left(I_{n}-T_{n,+}^{2}\right)\right)^{\frac{1}{n}}$ is an increasing subsequence for $n=3,5,7, \ldots$ and a decreasing subsequence for $n=4,6,8, \ldots$ Both subsequences converge to $\left(\frac{1+\sqrt{5}}{2}\right)^{2} \approx 2.6180$. Hence

$$
\left(\operatorname{det}\left(I_{n}-T_{n,+}^{2}\right)\right)^{\frac{1}{n}} \leqslant(20)^{\frac{1}{3}} \approx 2.7144 \text { for } n \geqslant 5 .
$$

Equality holds if and only if $n=6$.

Proof. Let $S$ be a skew symmetric matrix and $a>0$. Then

$$
\begin{aligned}
& \operatorname{det}\left(a^{2} I-S^{2}\right)=\operatorname{det}((a I-S)(a I+S))=\operatorname{det}(a I+S) \operatorname{det}(a I-S) \\
& =\operatorname{det}(a I+S) \operatorname{det}\left((a I-S)^{\top}\right)=\operatorname{det}(a I+S)^{2} .
\end{aligned}
$$


Hence

$$
\operatorname{det}\left(I_{n}-T_{n,+}^{2}\right)=\left(\operatorname{det}\left(I_{n}+T_{n,+}\right)\right)^{2}, \quad \operatorname{det}\left(I_{n}+T_{n,-}^{2}\right)=\left(\operatorname{det}\left(I_{n}-T_{n,-}\right)\right)^{2} .
$$

Next we claim that

$$
\operatorname{det}\left(I_{n}+t T_{n,-}\right)=\sum_{j=0}^{\left\lceil\frac{n}{2}\right\rceil-1} \phi\left(j, C_{n}\right) t^{2 j} .
$$

First observe that since $\operatorname{det} T_{n,+}=0$ the polynomial $\operatorname{det}\left(I_{n}+t T_{n,-}\right)$ is of degree $n-1$ at most. Let $k \in[n-1]$. Then the coefficient of $t^{k}$ is the sum of all $\operatorname{det} T_{n,-}[J]$ where $J$ is a a subset of cardinality of $k$ of $[n]$. As $T_{n,-}[J]$ represents an orientation of a subgraph $C_{n}[J]$, which is a forest. As any orientation of the forest is Pfaffian we deduce that $\operatorname{det} C_{n}[J]$ is a square of the number of perfect matches in the subgraph $C_{n}[J]$. Observe next a forest has either 0 or 1 perfect match. Hence $\operatorname{det} C_{n}[J]$ is the number of perfect matches in the subgraph $C_{n}[J]$. Thus $\sum_{|J|=k} \operatorname{det} T_{n,-}[J]$ is zero if $k$ is odd, and $\phi\left(\frac{k}{2}, C_{n}\right)$ if $k$ is even. Since $\operatorname{det} I_{n}=1$ we deduce (6.7).

Next we claim that

$$
\begin{cases}\operatorname{det}\left(I_{n}+t T_{n,+}\right)=\operatorname{det}\left(I_{n}+t T_{n,-}\right) & \text { if } n \text { odd } \\ \operatorname{det}\left(I_{n}+t T_{n,+}\right)=\operatorname{det}\left(I_{n}+t T_{n,-}\right)+4 t^{n} & \text { if } n \text { even }\end{cases}
$$

Indeed, the above arguments show that $\operatorname{det}\left(I_{n}+t T_{n,+}\right)-\operatorname{det}\left(I_{n}+t T_{n,-}\right)=t^{n} \operatorname{det} T_{n,+}$. Hence (6.8) holds. Let $\phi\left(t, C_{n}\right):=\sum_{j=0}^{\left\lfloor\frac{n}{2}\right\rfloor} \phi\left(j, C_{n}\right) t^{2 j}$ be the matching polynomial of $C_{n}$. Combine (6.7) and (6.8) to deduce

$$
\begin{cases}\operatorname{det}\left(I_{n}+t T_{n,+}\right)=\phi\left(t, C_{n}\right) & \text { if } n \text { odd } \\ \operatorname{det}\left(I_{n}+t T_{n,+}\right)=\phi\left(t, C_{n}\right)+2 t^{n} & \text { if } n \text { even }\end{cases}
$$

Let $P_{n}$ be a path of length $n$. Denote by $\phi\left(t, P_{n}\right)$ the matching polynomial of a path of length $n$. Then the following identities are straightforward, see $[9,(2.5),(2.6)]$ :

$$
\begin{aligned}
& \phi\left(t, P_{n}\right)=\phi\left(t, P_{n-1}\right)+t \phi\left(t, P_{n-2}\right), \\
& \phi\left(t, C_{n}\right)=\phi\left(t, P_{n}\right)+t \phi\left(t, P_{n-2}\right) .
\end{aligned}
$$

It easily follows that $\phi\left(1, P_{n}\right)$ is the standard Fibonacci sequence for $1,1,2,3,5, \ldots$ for $n=0,1,2,3,4, \ldots$. Use this fact and (6.11) to obtain

$$
\phi\left(1, C_{n}\right)=\left(\frac{1+\sqrt{5}}{2}\right)^{n}+\left(\frac{1-\sqrt{5}}{2}\right)^{n} \text { for } n \geqslant 3 .
$$
Let

Combine (6.12), (6.9), (6.8) and (6.6) to deduce (6.4). Denote $\epsilon:=\frac{\sqrt{5}-1}{\sqrt{5}+1}, \delta:=\frac{2}{\sqrt{5}+1}$. $a_{n}:=\left(\operatorname{det}\left(I_{n}-T_{n,+}^{2}\right)\right)^{\frac{1}{n}}, b_{n}:=\left(\frac{\sqrt{5}+1}{2}\right)^{2}\left(1-\epsilon^{n}\right)^{\frac{2}{n}}, c_{n}:=\left(\frac{\sqrt{5}+1}{2}\right)^{2}\left(1+\epsilon^{n}+2 \delta^{n}\right)^{\frac{2}{n}}, n \in \mathbb{N}$. 
Then this subsequence is equal to the subsequence for $n=3,5,7, \ldots$. Then $a_{n}=b_{n}$ for odd $n$ and $a_{n}=c_{n}$ for an even $n$. As $\epsilon, \delta \in(0,1)$ we deduce

$$
\begin{aligned}
& \left(1-\epsilon^{n}\right)^{\frac{1}{n}}<\left(1-\epsilon^{n+1}\right)^{\frac{1}{n}}<\left(1-\epsilon^{n+1}\right)^{\frac{1}{n+1}} \\
& \left(1+\epsilon^{n}+2 \delta^{n}\right)^{\frac{1}{n}}>\left(1+\epsilon^{n+1}+2 \delta^{n+1}\right)^{\frac{1}{n}}>\left(1+\epsilon^{n+1}+2 \delta^{n+1}\right)^{\frac{1}{n+1}}
\end{aligned}
$$

Hence $a_{n}, n=1,3, \ldots$ is an increasing sequence and $a_{n}, n=2,4, \ldots$, is a decreasing sequence which both converge to $\left(\frac{\sqrt{5}+1}{2}\right)^{2}$. Therefore inequality (6.5) holds.

Theorem 19. Let $G=(V, E), n=|E|$ be a semi-circular cubic graph where $n_{1}=\left|V_{1}\right|$ and $n_{0}=\left|V_{0}\right|$ is the number of vertices of $G$ in the the closed disk bounded by the first circle $O_{1}$ and its interior respectively. If $G$ does not have 3 and 4-cycles then

$$
\begin{array}{cl}
\text { perfmat } G \leqslant 20^{\frac{n-n_{1}}{12}} 3^{\frac{n_{1}}{4}} & \text { if } n_{0}>0, \\
\text { perfmat } G \leqslant 20^{\frac{n}{12}} & \text { if } G \text { is circular, i.e. } n_{0}=0 .
\end{array}
$$

Proof. Note that if $G$ is a semi-circular cubic graph then each vertex on a circle $O_{i}$ for $i>1$ has exactly one edge which connects it either to $O_{i-1}$ or to $O_{i+1}$. Hence (6.3) holds. Clearly $\operatorname{det} B\left[V_{1}\right] \leqslant 3^{\frac{n_{1}}{3}}$. Use Lemma 18 to deduce (6.13). Assume now that $n_{0}=0$, i.e. $G$ is circular. Then the arguments of the proof of Proposition 17 yields that $\operatorname{det} B\left[V_{1}\right] \leqslant \max \left(\operatorname{det}\left(I_{m_{1}}+T_{m_{1},+}^{2}\right), \operatorname{det}\left(I_{m_{1}}+T_{m_{1},-}^{2}\right)\right)$, where $\left|V_{1}\right|=n_{1}=m_{1}$. Lemma 18 yields that $\operatorname{det} B\left[V_{1}\right] \leqslant 20^{\frac{m_{1}}{3}}$. Hence (6.14) holds.

Since $20^{\frac{1}{3}} \approx 2.7144<\sqrt{8} \approx 2.8284$ we deduce that $(6.14)$ is better than the best estimate $8^{\frac{n}{8}}$ for fullerene graphs with hamiltonian cycle given in (5.2).

In applying Theorem 19 to fullerene graphs one needs to have a good estimate on $n_{1}$. The examples that we considered suggest that there exists a universal constant $N_{1}$ such that any fullerene graph is a semi-circular graph with $\left|V_{1}\right| \leqslant N_{1}$.

The next obvious problem is how good is the upper bound (6.14) for circular fullerene graph. Recall that a fullerene with nontrivial cyclic 5 -edge cut one has at least $5^{\frac{n-20}{10}}$ perfect matches $[18]$ and $[16,8-4]$.

\section{References}

[1] N. Alon and S. Friedland, The maximum number of perfect matchings in graphs with a given degree sequence, with N. Alon, The Electronic Journal of Combinatorics, 15 (2008), \#13, 1-2.

[2] G. Brinkmann, J. Goedgebeur and B. D. McKay, The generation of fullerenes, http: //arxiv.org/pdf/1207.7010.pdf.

[3] M. Chudnovsky and P. Seymour, Perfect matchings in planar cubic graphs, Combinatorica, 32 (2012) 403-424.

[4] P. Erdős and A. Rényi, On random matrices, II, Studia Sci. Math. Hungar. 3 (1968), 459-464. 
[5] L. Esperet, F. Kardos, A. King, D. Kral and S. Norine, Exponentially many perfect matchings in cubic graphs, Advances in Mathematics 227 (2011), 1646-1664.

[6] P.W. Fowler and D.B. Redmond, Symmetry aspects of leapfrog and truncated polyhedra, Match Communications in Mathematical and in Computer Chemistry 33 (1996), $101-119$.

[7] S. Friedand, A lower bound for the permanent of doubly stochastic matrices, Ann. of Math. 110 (1979), 167-176.

[8] S. Friedland and S. Gaubert, Submodular spectral functions of principal submatrices of an hermitian matrix, Linear Algebra and its Applications, 2012, available on line, arXiv:1007.3478.

[9] S. Friedland, E. Krop and K. Markström, On the Number of Matchings in Regular Graphs, The Electronic Journal of Combinatorics, 15 (2008), \#R110, 1-28, arXiv:0801.2256.

[10] P.W. Kasteleyn, Graph theory and crystal physics, in Graph Theory and Theoretical Physics (ed. by F. Harary), Academic Press, New York 1967, 43-110.

[11] F. Kardos, D. Král, J. Miškuf and J.-S. Sereni, Fullerene graphs have exponentially many perfect matchings, Journal of Mathematical Chemistry 46, 2 (2009) 443-447

[12] F. Kardos and R. Škrekovski, Cyclic edge-cuts in fullerene graphs, J. Mathe- matical Chemistry, 44 (2008), 121-132.

[13] D. Král, O. Pangrác, J.-S. Sereni and R. Škrekovski, Long cycles in fullerene graphs, Journal of Mathematical Chemistry 45, (2009), 1021-1031.

[14] K. Kutnar and D. Marušič, On cyclic edge-connectivity of fullerenes. Discrete Applied Mathematics, 156 (2008), 1661-1669.

[15] J. Lehel, Generating all 4-regular planar graphs from the graph of octahedron, J.Graph Theory 5 (1981), 423-426.

[16] M. Loebl and J.-S. Sereni, Lecture 8, http://www. liafa.univ-paris-diderot.fr/ sereni/Lectures/GC_Spring09/gc09_8.pdf.

[17] L. Lovász and M.D. Plummer, Matching theory, Elsevier Science, Amsterdam, 1986.

[18] J. Qian and F. Zhang, On the number of Kekulé structures in capped zigzag nanotubes, J. Mathematical Chemistry, 38 (2005), 233-246, 2005.

[19] R. Thomas, A survey of Pfaffian orientations of graphs, Proceedings of the International Congress of Mathematicians, vol 3, pp. 963-984, Madrid, Spain, 2006.

[20] M. Voorhoeve, A lower bound for the permanents of certain (0,1)-matrices, Nederl. Akad. Wetensch. Indag. Math. 41 (1979) 83-86. 